\title{
X-ray and UV Spectra of Glycine within Coupled Cluster Linear Response Theory
}

\author{
Myhre, Rolf H.; Coriani, Sonia; Koch, Henrik
}

Published in:

Journal of Physical Chemistry Part A: Molecules, Spectroscopy, Kinetics, Environment and General Theory

Link to article, DOI:

10.1021/acs.jpca.9b06590

Publication date:

2019

Document Version

Peer reviewed version

Link back to DTU Orbit

Citation $(A P A)$ :

Myhre, R. H., Coriani, S., \& Koch, H. (2019). X-ray and UV Spectra of Glycine within Coupled Cluster Linear Response Theory. Journal of Physical Chemistry Part A: Molecules, Spectroscopy, Kinetics, Environment and General Theory, 123(45), 9701-9711. https://doi.org/10.1021/acs.jpca.9b06590

\section{General rights}

Copyright and moral rights for the publications made accessible in the public portal are retained by the authors and/or other copyright owners and it is a condition of accessing publications that users recognise and abide by the legal requirements associated with these rights.

- Users may download and print one copy of any publication from the public portal for the purpose of private study or research.

- You may not further distribute the material or use it for any profit-making activity or commercial gain

- You may freely distribute the URL identifying the publication in the public portal 


\title{
X-ray and UV Spectra of Glycine within Coupled Cluster Linear Response Theory
}

\author{
Rolf H. Myhre, ${ }^{\dagger, \S}$ Sonia Coriani, ${ }^{*,+, \|}$ and Henrik Koch ${ }^{*, \dagger, \uparrow}$ \\ $\dagger$ Department of Chemistry, Norwegian University of Science and Technology, NTNU, 7491 \\ Trondheim, Norway \\ $\ddagger D T U$ Chemistry, Technical University of Denmark, DK-2800 Kongens Lyngby, Denmark \\ 9Scuola Normale Superiore, Piazza dei Cavaleri 7, 56126 Pisa, Italy \\ $\S$ Hylleraas Centre for Quantum Molecular Sciences, Department of Chemistry, University \\ of Oslo, 0315 Oslo, Norway \\ ||Aarhus Institute of Avanced Studies, University of Aarhus, DK-8000 Arhus C, Denmark \\ E-mail: soco@kemi.dtu.dk; henrik.koch@sns.it
}




\begin{abstract}
The coupled cluster models CCSD and CC3 are used to investigate the (core) excited states and ionization energies of glycine in the gas phase. Excited states and ionization energies in the UV spectral range are calculated using standard coupled cluster linear response, while core-level excited states and ionization potentials are calculated using the core-valence separation approximation. The temperature dependence from different conformers is also assessed.
\end{abstract}

\title{
Introduction
}

Glycine is the smallest amino acid found in proteins and is often used as a model system for studying properties of biological molecules. ${ }^{1}$ Due to its essential role in living organisms, there have been numerous studies trying to detect glycine in the interstellar medium as this would support the hypothesis of panspermia. ${ }^{2-6}$ Even though claims of its successful detection in three high-mass star-formation regions were made in $2003,{ }^{2}$ they were disputed just two years later ${ }^{3}$ and finally rejected in $2007 .^{4,5}$ Thus, glycine in the interstellar medium remains elusive, despite being one of the most intensively hunted prebiotic molecules.

In the gas phase glycine exists as neutral species while in solution it is a zwitterion. Glycine is also one of the smallest molecules with internal hydrogen bonding, which results in a large number of stable conformers. ${ }^{7-9}$ The literature contains many experimental studies on glycine characterizing the properties of the molecule, including several X-ray spectroscopy studies in gas phase,${ }^{10-12}$ in solution ${ }^{13}$ and in solid state. ${ }^{14,15}$ However, gas phase UV absorption spectra have not been reported so far, probably due to the low vapor pressure making such an experiment challenging.

Accurate computational methods can be an invaluable help when interpreting experimental results such as X-ray absorption spectra. ${ }^{16}$ The static exchange (STEX) method ${ }^{17}$ has been used in many studies to simulate spectra. While STEX can give good qualitative spectra, it requires shifts of several $\mathrm{eV}$ to agree with experimental peaks. A more advanced 
method is the Algebraic Diagrammatic Construction (ADC). ${ }^{18,19}$ In this approach, an effective Hamiltonian is constructed based on perturbation theory. This results in a size extensive and explicitly Hermitian theory. By combining this method with the core-valence separation (CVS) approximation, ${ }^{20-22}$ the $\mathrm{ADC}(2)$ approach has often been used to calculate X-ray absorption spectra. For instance, Wenzel et al. ${ }^{23}$ were able to calculate X-ray absorption spectra at the $\mathrm{ADC}(2)$ and $\mathrm{UADC}(2)$ level of medium sized molecules. The extended $\mathrm{ADC}(2)$ method, named $\mathrm{ADC}(2)-\mathrm{x}$, showed good agreement with experimental data, but fortuitous error cancellation probably plays an important role, and the accuracy is difficult to improve systematically. ${ }^{24}$

Coupled cluster (CC) theory ${ }^{25-28}$ is one the of the most accurate electronic structure models routinely used in quantum chemistry. Applying linear response (LR) ${ }^{29,30}$ or equation of motion (EOM) theory, ${ }^{31,32}$ it is possible to quantitatively predict molecular properties such as absorption and ionization spectra. However, using CC theory to simulate X-ray spectra has been lagging behind for a long time, due to the difficulties to selectively converge to the high energetic core states and to recover the large relaxation effects that occur upon promotion of a core electron.

An early attempt to address core excitations with CC theory is the two step open shell electron attachment (OS-EA-EOMCC) procedure of Nooijen and Bartlett, that utilized a coupled cluster singles and doubles (CCSD) description of the core ion to include the large relaxation effects, followed by adding an extra electron to the core-ionized state to obtain the various core-excited states of the neutral system. ${ }^{33}$ More than a decade later, Lanczos and Arnoldi based procedures were explored, ${ }^{34-36}$ as well as state specific multireference $\mathrm{CC},{ }^{37}$ complex polarization propagator, ${ }^{38}$ restricted window ${ }^{39}$ and time dependent EOMCC $^{40}$ schemes.

The CVS approximation was introduced in CCLR/EOMCC in 2015 by Coriani and $\operatorname{Koch}^{41,42}$ in the form of a projector operator that iteratively eliminated pure valence excitations during the solution of the $\mathrm{CC}$ eigenvalue equations. By removing the space of valence 
excited states from the solution space, convergence problems are avoided. Using CVS has proven extremely efficient in yielding core excitations and ionisations within CCLR and EOMCC theory. ${ }^{43-47}$ In general, calculation of spectroscopic observables related to the interaction of the molecular system with X-ray radiation displays strong dependence on the level of theory and size of the basis set, especially when describing excited states with Rydberg character. ${ }^{44,45,47,48}$

Because core excitations and ionizations tend to be highly localized, they are well suited for local approaches. In multilevel $\mathrm{CC},{ }^{49}$ a part of the molecule is treated with higher accuracy than the rest in order to reduce computational costs. The multilevel CC method successfully described X-ray spectra when combined with the CVS approximation. ${ }^{50-52}$ Another approach to reduce the cost of EOMCC is using a perturbative description of the excited states. By solving for the excited states in the much smaller space of singly excited states and then including correlation from the doubles perturbatively, Sadybekov and Krylov were able to model the X-ray spectra of glycine in aqueous solvent and predicted significant differences in the spectra between different protonated forms. ${ }^{53}$

The core-valence separation approximation has also been combined with the frozen core (fc) approximation for the ground state, ${ }^{48}$ yielding the fc-CVS-EOMCCSD method. Intuitively, one would expect errors from excluding correlation for the core orbitals in the ground state, but the approximation results in a constant shift of the whole core-excited spectrum, similar to the inclusion of core correlating functions in the basis set, ${ }^{45}$ except in the opposite direction. This can be understood as the improved description from the core orbitals lowering the ground state energy while the lack of correlation from the frozen core raising it. Because CVS-CCSD consistently overestimates core excitation and ionization energies, the fc-CVS-CCSD core excitations and ionization energies were found to be systematically closer to the experimental reference data than the CVS-CCSD ones, whereas the spectral intensities are basically the same.

Very recently, the CVS approximation has also been combined with the $\triangle \mathrm{CC}$ method 
and successfully used to describe core ionizations. ${ }^{54}$ In this method, CC states are computed using wavefunctions with core holes as reference and CVS is used to ensure the method does not collapse towards the ground state.

In this work, we present and compare spectroscopic data of glycine calculated at the $\mathrm{CCSD}^{27}$ and CC3 (coupled cluster singles, doubles and approximate triples) ${ }^{28,50}$ levels of theory. Specifically, we have modelled absorption and ionization spectral parameters in the UV and X-ray energy ranges using the LR formalism, and compared them to experimental values from the literature. ${ }^{11,12}$ We also compare our CCSD results with the results obtained with the fc-CVS-CCSD approach. As excited state properties show a strong dependence on the basis set, we have performed calculations with different sets in order to determine the best compromise between accuracy and cost. We also investigate the effect of different conformers.

\section{Theory}

In coupled cluster theory, the wave function is expressed as the exponential of a cluster operator, $T$, operating on a reference state, typically a Hartree-Fock (HF) wavefunction.

$$
|\mathrm{CC}\rangle=\exp (T)|\mathrm{HF}\rangle
$$

The cluster operator is a sum of excitation operators $\left(\tau_{\mu}\right)$ each weighted by the corresponding

amplitude $\left(t_{\mu}\right), T=\sum_{\mu} t_{\mu} \tau_{\mu}$, where $\mu$ refers to excited determinants in the Fock space. The wave function parameters are then determined by projection.

$$
\begin{aligned}
E & =\langle\mathrm{HF}|\exp (-T) H \exp (T)| \mathrm{HF}\rangle \\
\Omega_{\mu} & =\langle\mu|\exp (-T) H \exp (T)| \mathrm{HF}\rangle=0
\end{aligned}
$$

Excitation energies and excited state properties are usually calculated using either CC 
linear response $(\mathrm{LR})^{29,30}$ or equation of motion $(\mathrm{EOM})$ CC. ${ }^{32}$ The critical step in both methods is finding the eigenvalues of the Jacobian matrix, $\boldsymbol{A}$, with elements given by eq. (3).

$$
A_{\mu \nu}=\frac{\partial \Omega_{\mu}}{\partial t_{\nu}}=\left\langle\mu\left|\exp (-T)\left[H, \tau_{\nu}\right] \exp (T)\right| \mathrm{HF}\right\rangle
$$

Because the Jacobian is non-symmetric, both the right, $\boldsymbol{R}_{i}$, and left, $\boldsymbol{L}_{i}$ eigenvectors must be determined in order to obtain excited state properties

$$
\boldsymbol{A} \boldsymbol{R}_{i}=\omega_{i} \boldsymbol{R}_{i} \quad \boldsymbol{L}_{i}^{T} \boldsymbol{A}=\omega_{i} \boldsymbol{L}_{i}^{T}
$$

The eigenvectors satisfy the biorthogonality condition, $\boldsymbol{L}_{i}^{T} \boldsymbol{R}_{j}=\delta_{i j}$, and the eigenvalues, $\omega_{i}$, are the excitation energies of the system.

We employ the Davidson algorithm ${ }^{55}$ to determine the eigenvalues. This algorithm constructs a subspace, starting with the lowest eigenvalue. As a result, finding eigenvalues corresponding to core excited states is not straightforward because they are embedded in a continuum of valence excited states. The CVS approximation ${ }^{20,41}$ overcomes this limitation by taking advantage of the very small coupling between valence- and core excited states. Vector elements corresponding to such couplings can then be set to zero without loss of accuracy and the algorithm will not collapse to valence excited states.

In order to calculate ionization energies, an extremely diffuse orbital can be added to the basis set. ${ }^{41,56}$ Integrals involving this orbital will then be zero and ionization energies are approximated as the states with excitations to this orbital. It is possible to estimate the (relative) strength of the corresponding ionization using the norm of the Dyson orbitals, ${ }^{57}$ which can be obtained from the transition density matrix elements for excitations to and from the diffuse orbital. ${ }^{58}$ However, we have not calculated the strengths in this work. 


\section{Results}

\section{Geometries}

In order to compare our results to experiment, accurate geometries are required. Miller and Clary $^{1}$ identified five important conformers of glycine, summarized in Figure 1. Conformer I indicates the numbering scheme adopted in this work, where atoms are ordered after their 1s orbital energy in descending order.
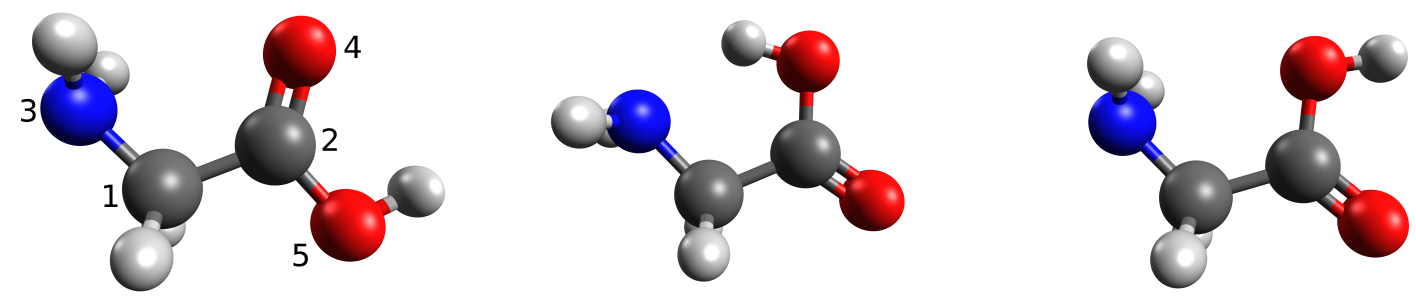

$\mathrm{I}\left(\mathrm{E}=-283.80990 \mathrm{E}_{\mathrm{h}}\right)$

II $(\Delta \mathrm{E}=+3.29 \mathrm{~kJ} / \mathrm{mol})$

III $(\Delta \mathrm{E}=+6.89 \mathrm{~kJ} / \mathrm{mol})$
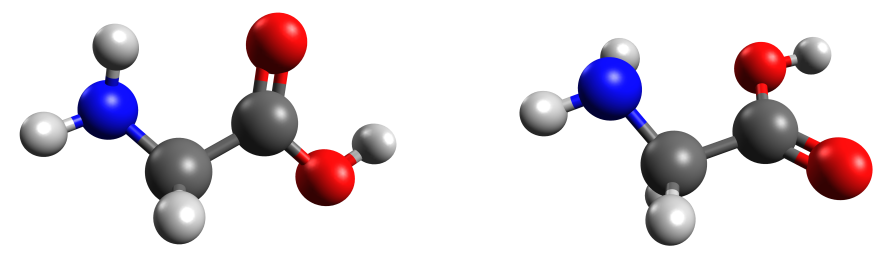

IV $(\Delta \mathrm{E}=+5.46 \mathrm{~kJ} / \mathrm{mol})$

$\mathrm{V}(\Delta \mathrm{E}=+10.21 \mathrm{~kJ} / \mathrm{mol})$

Figure 1: Conformers of glycine with energies calculated at the CCSD(T)/aug-cc-pVDZ level. For conformer I, the total energy is indicated in atomic units. The relative energies with respect to conformer $\mathrm{I}$ in $\mathrm{kJ} / \mathrm{mol}$ are reported for the other conformers.

The geometries were optimized at the $\operatorname{CCSD}(\mathrm{T})$ level using $\mathrm{CFOUR}^{59,60}$ and the augcc-pVDZ basis set. ${ }^{61}$ A larger basis set would have been preferable, especially the cc-pVTZ basis set because it shows favorable error cancellation with $\operatorname{CCSD}(\mathrm{T})$, but this would have been computationally too expensive. ${ }^{62}$ Judging from the relatively small differences between the different conformers, this is unlike to cause noticeable errors.

Zero-point vibrational energies were also calculated using the same model and basis set. This was also done in CFOUR using the harmonic approximation and analytic second derivatives. ${ }^{63}$ The zero point energies for all conformers were about $2.15 \mathrm{eV}$, consistent with 
earlier studies. ${ }^{1}$ Geometries, ground state energies and zero point energies are collected in the Supplementary Information file. The optimized geometries of conformers I, II and III have $\mathrm{C}_{s}$ symmetry up to the convergence threshold and most states could be clearly assigned as either $\mathrm{A}^{\prime}(\sigma$ character$)$, or $\mathrm{A}^{\prime \prime}$ ( $\pi$ character).

\section{UV excitation- and binding energies of conformer I}

In this section we present CCSD and CC3 UV excitation- and ionization energies calculated for the lowest energy conformer (conformer I) using various basis sets. We also present CCSD oscillator strengths computed in the length gauge. Our results are compared to experimental values when these are available.

Based on our experience, ${ }^{44}$ diffuse basis functions ${ }^{61}$ are important for excited state properties and should be included in all basis sets. Furthermore, core-valence polarization functions ${ }^{64}$ are required for accurate absolute core excitation energies. For the sake of brevity, we adopt a shorthand notation for the basis sets. For example, aug-cc-pCVDZ will be referred to as aCDZ. We also use mixed basis sets for core excitations and ionizations, and these are indicated with parentheses, for example, a(CT)Z means aug-cc-pCVTZ on the atom being excited and aug-cc-pVDZ on the rest.

Table 1 collects the results for the valence excitations and ionizations investigated. For the most expensive calculation, namely $\mathrm{CC} 3 / \mathrm{aCTZ}$, we were only able to compute four excited states due to the high computational cost. At least up to $7.5 \mathrm{eV}$, the differences in excitation energies between aCDZ and $\mathrm{aCTZ}$ are $0.2 \mathrm{eV}$ or less. However, when going from aCDZ to aCTZ with CC3, the order of the third and fourth excitations changes. The $\mathrm{A}^{\prime \prime}$ states involve orbitals with $\pi$ character, that extends further away from the atoms in the molecule, so it is not surprising that these are described better and get lower in energy relative to the $\mathrm{A}^{\prime}$ states when the basis set is increased with more diffuse orbitals. A similar, but less pronounced effect is observed for CCSD with the third and fourth states becoming almost degenerate with aCTZ. In general, larger basis sets increase the excitation energies, while going from 
CCSD to $\mathrm{CC} 3$ reduces them, indicating that there is some error cancellation at work. We note that Osted et al. ${ }^{65}$ have also investigated the UV excitations of (among others) glycine at the CCSD level of theory. Using the aug-cc-pVTZ basis set on the nitrogen atom and aug-cc-pVDZ on the rest, they reported $5.88 \mathrm{eV}\left(f=5.3 \times 10^{-4}\right), 6.32 \mathrm{eV}(+0.44 \mathrm{eV}$, $\left.f=1.25 \times 10^{-2}\right), 7.24 \mathrm{eV}\left(+1.36 \mathrm{eV}, f=4.61 \times 10^{-2}\right), 7.48 \mathrm{eV}\left(+1.60 \mathrm{eV}, f=1.54 \times 10^{-2}\right)$ and $7.61 \mathrm{eV}\left(+1.73 \mathrm{eV}, f=3.16 \times 10^{-2}\right)$ for their minimum energy conformer, similar to our results

As noted in the introduction, no experimental gas-phase UV results seem to be available in the literature. Nonetheless, we report in Figure 2 a simulated UV spectrum of conformer I calculated at the CCSD/aug-cc-pCVDZ level of theory. The computed sticks have been broadened with a Lorentzian line-shape function and a line broadening of $0.1 \mathrm{eV}$ half width at half maximum (HWHM). In order to simulate the whole spectrum up to the ionization edge, we used the aCDZ basis set in the calculations.

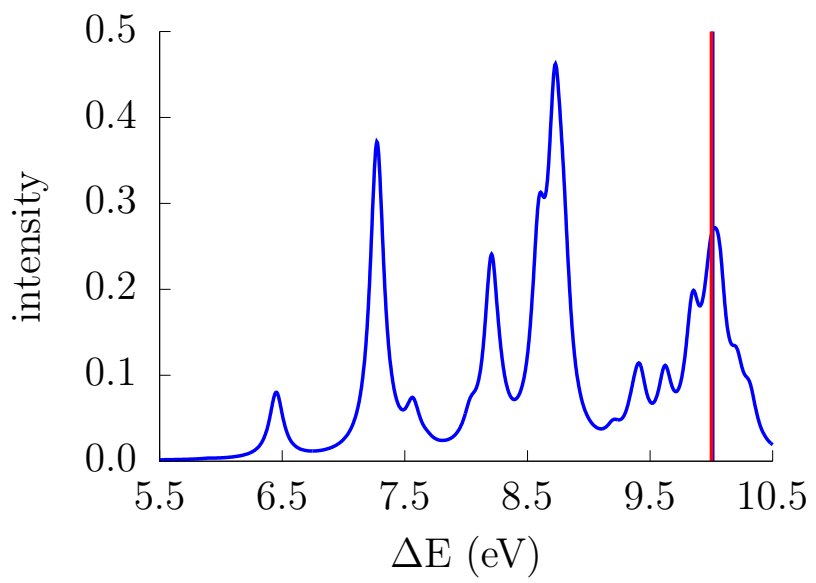

Figure 2: Simulated UV/Vis spectrum for conformer I using CCSD/aug-cc-pCVDZ with arbitrary intensity units. Vertical bars indicate the first CCSD/aCDZ (blue) and experimental (red) ionization energies (almost identical).

For the ionizations, the effect of changing the basis set is marginally larger, with the smaller basis set in a few instances yielding results slightly closer to the experimental ones. The deviations from the experimental values for both $\mathrm{CC}$ methods are in any case modest and do not exceed $0.32 \mathrm{eV}$. Similarly to the excitation energies, increasing the basis set tends 
to increase the ionization energies, whereas going from CCSD to CC3 reduces them.

Table 1: Calculated CCSD and CC3 excitation and ionization energies (eV), CCSD transition moments $(\times 100)$ and experimental ionization energies $( \pm 0.1)^{12}$ for conformer I. The higher excitations/ionizations are given relative to the first.

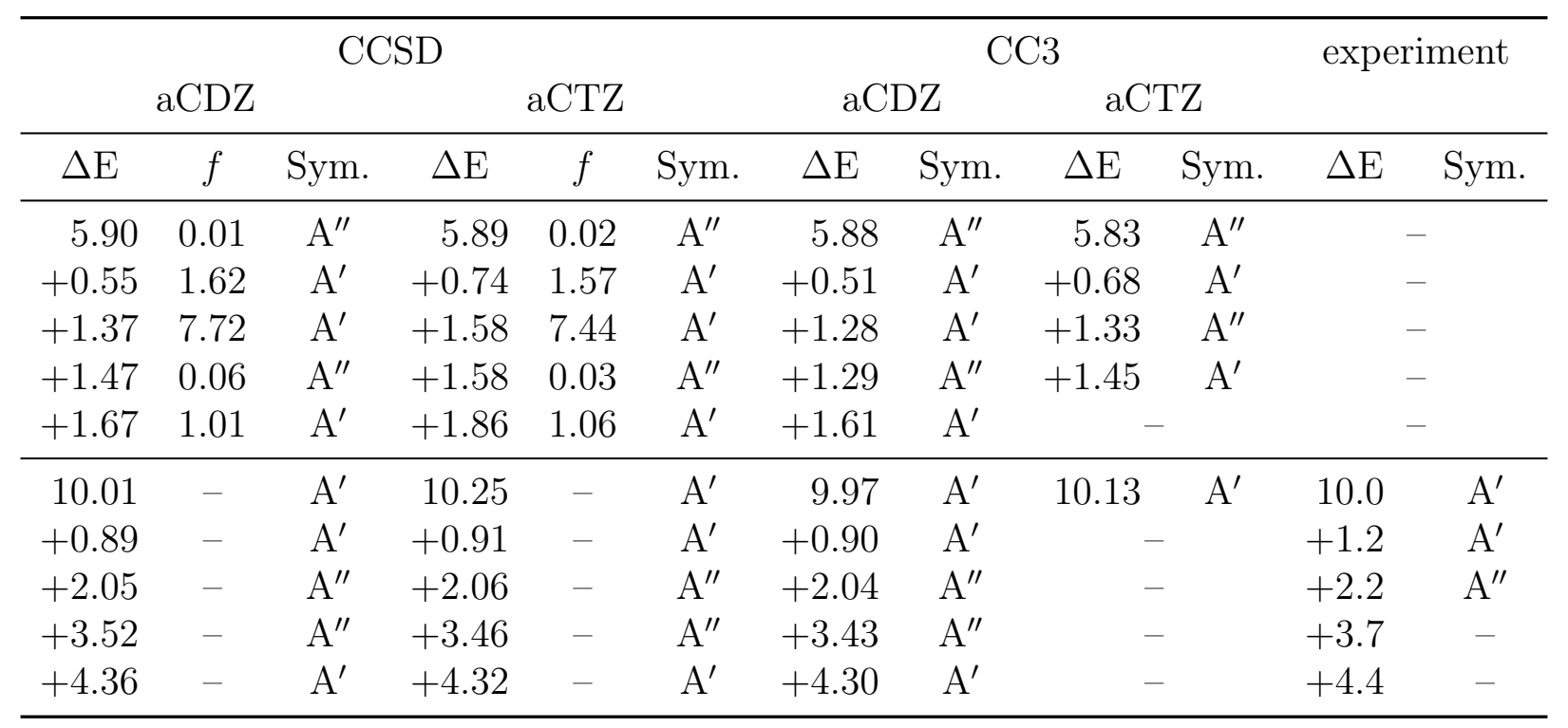

\section{Core excitation- and binding energies of conformer I}

We now turn our attention to the X-ray spectra of conformer I. The CVS-CCSD core excitation energies at all K-edges together with their relative intensities are presented in Table 2, whereas CVS-CC3 and experimental excitation energies are given in Table 3. In this section, CCSD will refer to CVS-CCSD-LR unless otherwise specified and CC3 will always refer to CVS-CC3LR. Notice that the results presented here do not take relativistic effects into account. Relativistic effects typically result in a constant shift of the whole spectrum, depending almost exclusively on the type of core. Including scalar relativistic effects would result in a shift of about $+0.10 \mathrm{eV}$ for carbon, $+0.21 \mathrm{eV}$ for nitrogen and $+0.39 \mathrm{eV}$ for oxygen. ${ }^{35,45,46}$

Comparing the results from different basis sets in Table 2, it is clear that the main effect of increasing the basis is a reduction of the excitation energy. This effect is largest for oxygen, where the difference is more than $2 \mathrm{eV}$, while it is closer to $1 \mathrm{eV}$ for carbon. The largest 
Table 2: CCSD core excitation energies (in $\mathrm{eV})$, corresponding intensities $(\times 100)$ and symmetries of conformer I. Higher excitation energies are given relative to the first.

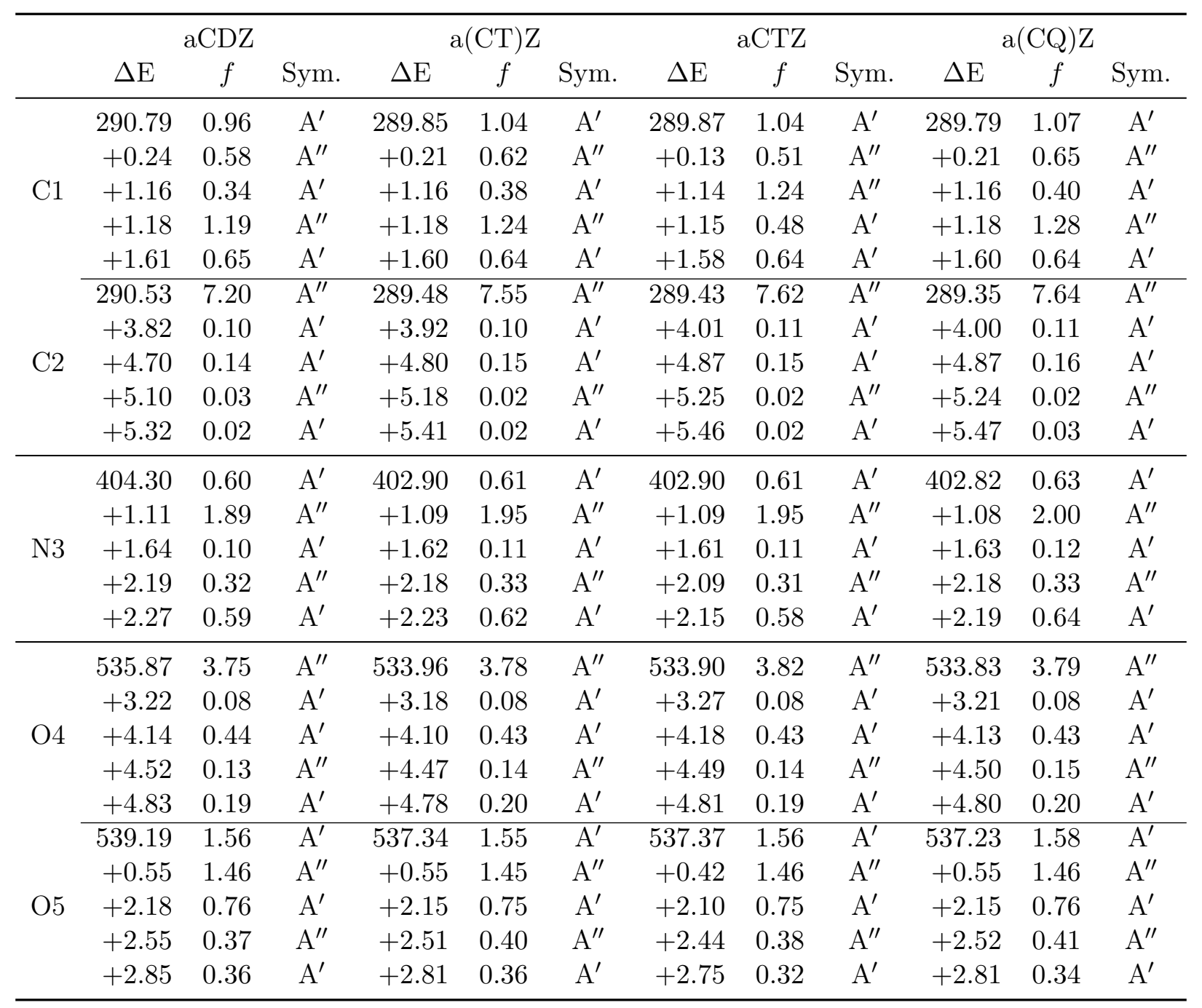


change is between aCDZ and $\mathrm{a}(\mathrm{CT}) \mathrm{Z}$ while increasing further to $\mathrm{aCTZ}$ or a(CQ)Z reduces the excitation energies with less than $0.15 \mathrm{eV}$.

While changing basis set results in large absolute shifts, the relative excitation energies change much less with no changes larger than $0.2 \mathrm{eV}$ and no change in the ordering of the states except the nearly degenerate third and fourth states for C1. Similarly, the changes in transition strength are also quite small, so a change in basis set will not result in large changes in the shape of the computed spectrum. We note that the largest change in relative positions and in intensity is observed for $\mathrm{C} 2$, especially the first excitation. This is a core excitation of the carbonyl $\mathrm{C}$ atom, and involves considerable charge transfer to oxygen.

In Table 4 we compare core excitations obtained using CVS-CCSDLR with fc-CVSEOMCCSD. The frozen core results are consistently just under $1 \mathrm{eV}$ lower than the standard results, due to the poorer description of the ground state. However, the relative positions are almost exactly the same and only minor differences are observed in the transition moments, probably due to the difference between LR and EOM, and the largest difference is observed for the first excitation of $\mathrm{C} 2$.

In Table 3 we present $\mathrm{CC} 3$ core excitation energies as well as experimental results from Ref. 11. Where appropriate, we have aligned the computed excitation energies with the experimental ones and we will discuss them for each atom. We also present simulated and experimental spectra for the different K-edges in Figures 3, 4, and 5. There are two versions of each spectrum, one with the calculated CCSD and CC3 excitation energies and one shifted. For nitrogen and oxygen, the simulated spectra are shifted to align the first peak in the experimental spectrum with the first peak in the simulated spectrum. For carbon, the position of the first experimental peak is unclear, see below, so we have shifted the spectrum to align with the intense $\mathrm{C} 2$ peak. Intensities in the spectra are normalized to match the most intense peak and the lines are broadened using a Lorentzian with HWHM maximum of $0.22 \mathrm{eV}$.

In Table 5, we have listed the difference between the two carbon K-edges and the two 
Table 3: Conformer I. CC3 core excitation energies and experimental results from Plekan et al. ${ }^{11}(\mathrm{eV})$. The point group symmetry could not be determined for the degenerate states of $\mathrm{C} 1$ with aCDZ. Higher excitation energies are given relative to the first.

\begin{tabular}{|c|c|c|c|c|c|c|c|c|c|}
\hline & $\mathrm{aCDZ}$ & Sym. & $\mathrm{a}(\mathrm{CT}) \mathrm{Z}$ & Sym. & $\mathrm{aCTZ}$ & Sym. & $\mathrm{a}(\mathrm{CQ}) \mathrm{Z}$ & Sym. & $\exp$ \\
\hline C1 & $\begin{array}{r}289.77 \\
+0.01 \\
+0.97 \\
+1.07 \\
+1.29\end{array}$ & $\begin{array}{l}\mathrm{A}^{*} \\
\mathrm{~A}^{*} \\
\mathrm{~A}^{\prime} \\
\mathrm{A}^{\prime \prime} \\
\mathrm{A}^{\prime}\end{array}$ & $\begin{array}{r}288.71 \\
+0.02 \\
+1.00 \\
+1.08 \\
+1.31\end{array}$ & $\begin{array}{l}\mathrm{A}^{\prime \prime} \\
\mathrm{A}^{\prime} \\
\mathrm{A}^{\prime} \\
\mathrm{A}^{\prime \prime} \\
\mathrm{A}^{\prime}\end{array}$ & 288.59 & $\mathrm{~A}^{\prime \prime}$ & 288.57 & $\mathrm{~A}^{\prime \prime}$ & +1.7 \\
\hline $\mathrm{C} 2$ & $\begin{array}{r}290.15 \\
+2.93 \\
+3.71 \\
+4.20 \\
+4.32\end{array}$ & $\begin{array}{l}\mathrm{A}^{\prime \prime} \\
\mathrm{A}^{\prime} \\
\mathrm{A}^{\prime} \\
\mathrm{A}^{\prime \prime} \\
\mathrm{A}^{\prime}\end{array}$ & $\begin{array}{r}288.97 \\
+3.06 \\
+3.84 \\
+4.30 \\
+4.44\end{array}$ & $\begin{array}{l}\mathrm{A}^{\prime \prime} \\
\mathrm{A}^{\prime} \\
\mathrm{A}^{\prime} \\
\mathrm{A}^{\prime \prime} \\
\mathrm{A}^{\prime}\end{array}$ & 288.94 & $\mathrm{~A}^{\prime \prime}$ & 288.78 & $\mathrm{~A}^{\prime \prime}$ & 288.4 \\
\hline N3 & $\begin{array}{r}402.76 \\
+1.00 \\
+1.17 \\
+1.18 \\
+1.78\end{array}$ & $\begin{array}{l}\mathrm{A}^{\prime} \\
\mathrm{A}^{\prime \prime} \\
\mathrm{A}^{\prime} \\
\mathrm{A}^{\prime \prime} \\
\mathrm{A}^{\prime}\end{array}$ & $\begin{array}{r}401.34 \\
+1.02 \\
+1.20 \\
+1.20 \\
+1.81\end{array}$ & $\begin{array}{l}\mathrm{A}^{\prime} \\
\mathrm{A}^{\prime \prime} \\
\mathrm{A}^{\prime} \\
\mathrm{A}^{\prime \prime} \\
\mathrm{A}^{\prime}\end{array}$ & 401.34 & $\mathrm{~A}^{\prime}$ & 401.20 & $\mathrm{~A}^{\prime}$ & $\begin{array}{l} \\
+2.6 \\
+3.6\end{array}$ \\
\hline $\mathrm{O} 4$ & $\begin{array}{r}533.94 \\
+1.70 \\
+2.50 \\
+2.90 \\
+3.07\end{array}$ & $\begin{array}{l}\mathrm{A}^{\prime \prime} \\
\mathrm{A}^{\prime} \\
\mathrm{A}^{\prime} \\
\mathrm{A}^{\prime} \\
\mathrm{A}^{\prime}\end{array}$ & $\begin{array}{r}532.10 \\
+1.83 \\
+2.64 \\
+3.04 \\
+3.22\end{array}$ & $\begin{array}{l}\mathrm{A}^{\prime \prime} \\
\mathrm{A}^{\prime} \\
\mathrm{A}^{\prime} \\
\mathrm{A}^{\prime \prime} \\
\mathrm{A}^{\prime}\end{array}$ & 532.01 & $\mathrm{~A}^{\prime \prime}$ & 531.90 & $\mathrm{~A}^{\prime \prime}$ & 532.2 \\
\hline O5 & $\begin{array}{r}536.86 \\
+0.06 \\
+1.44 \\
+1.88 \\
+2.04\end{array}$ & $\begin{array}{l}\mathrm{A}^{\prime \prime} \\
\mathrm{A}^{\prime} \\
\mathrm{A}^{\prime} \\
\mathrm{A}^{\prime \prime} \\
\mathrm{A}^{\prime}\end{array}$ & $\begin{array}{r}535.16 \\
+0.06 \\
+1.49 \\
+1.92 \\
+2.08\end{array}$ & $\begin{array}{l}\mathrm{A}^{\prime \prime} \\
\mathrm{A}^{\prime} \\
\mathrm{A}^{\prime} \\
\mathrm{A}^{\prime \prime} \\
\mathrm{A}^{\prime}\end{array}$ & 535.01 & $\mathrm{~A}^{\prime \prime}$ & 534.99 & $\mathrm{~A}^{\prime \prime}$ & $\begin{array}{c}+7.0 \\
535.4\end{array}$ \\
\hline
\end{tabular}


Table 4: Comparison of excitation energies and intensities $(\times 100)$ between CVSCCSDLR and fc-CVS-EOMCCSD using the aCDZ basis set. Higher excitation energies are given relative to the first.

\begin{tabular}{|c|c|c|c|c|}
\hline & \multicolumn{2}{|c|}{ LR } & \multicolumn{2}{|c|}{ fc-EOM } \\
\hline & $\Delta \mathrm{E}$ & $f$ & $\Delta \mathrm{E}$ & $f$ \\
\hline \multirow{5}{*}{$\mathrm{C} 1$} & 290.79 & 0.96 & 289.81 & 0.97 \\
\hline & +0.24 & 0.58 & +0.23 & 0.69 \\
\hline & +1.16 & 0.34 & +1.16 & 0.35 \\
\hline & +1.18 & 1.19 & +1.18 & 1.21 \\
\hline & +1.61 & 0.65 & +1.61 & 0.66 \\
\hline \multirow{5}{*}{$\mathrm{C} 2$} & 290.53 & 7.20 & 289.55 & 7.50 \\
\hline & +3.82 & 0.10 & +3.82 & 0.11 \\
\hline & +4.70 & 0.14 & +4.70 & 0.15 \\
\hline & +5.10 & 0.03 & +5.11 & 0.03 \\
\hline & +5.32 & 0.02 & +5.32 & 0.02 \\
\hline \multirow{5}{*}{ N3 } & 404.30 & 0.60 & 403.33 & 0.62 \\
\hline & +1.11 & 1.89 & +1.11 & 1.93 \\
\hline & +1.64 & 0.10 & +1.64 & 0.10 \\
\hline & +2.19 & 0.32 & +2.19 & 0.33 \\
\hline & +2.27 & 0.59 & +2.27 & 0.59 \\
\hline \multirow{5}{*}{$\mathrm{O} 4$} & 535.87 & 3.75 & 534.90 & 3.91 \\
\hline & +3.22 & 0.08 & +3.22 & 0.09 \\
\hline & +4.14 & 0.44 & +4.15 & 0.45 \\
\hline & +4.52 & 0.13 & +4.52 & 0.13 \\
\hline & +4.83 & 0.19 & +4.84 & 0.19 \\
\hline \multirow{5}{*}{$\mathrm{O} 5$} & 539.19 & 1.56 & 538.23 & 1.60 \\
\hline & +0.55 & 1.46 & +0.55 & 1.52 \\
\hline & +2.18 & 0.76 & +2.18 & 0.76 \\
\hline & +2.55 & 0.37 & +2.55 & 0.38 \\
\hline & +2.85 & 0.36 & +2.85 & 0.36 \\
\hline
\end{tabular}

Table 5: Differences between the lowest excitation energies for each atom.

\begin{tabular}{llll}
\hline & & CCSD & CC3 \\
\hline \multirow{2}{*}{$\mathrm{C} 2-\mathrm{C} 1$} & $\mathrm{aCDZ}$ & -0.26 & +0.39 \\
& $\mathrm{aCTZ}$ & -0.45 & +0.35 \\
\hline \multirow{2}{*}{$\mathrm{O} 5-\mathrm{O} 4$} & $\mathrm{aCDZ}$ & +3.32 & +2.93 \\
& $\mathrm{aCTZ}$ & +3.46 & +3.00 \\
\hline
\end{tabular}


oxygen K-edges calculated with CCSD and CC3 using the aCDZ and aCTZ basis sets. The differences vary, especially between CCSD and CC3, demonstrating that the shape of a calculated spectrum might be wrong, even if the relative excitation energies for each atom are accurate. Note that there is virtually no difference between CVS-CCSDLR and fc-CVSEOMCCSD.

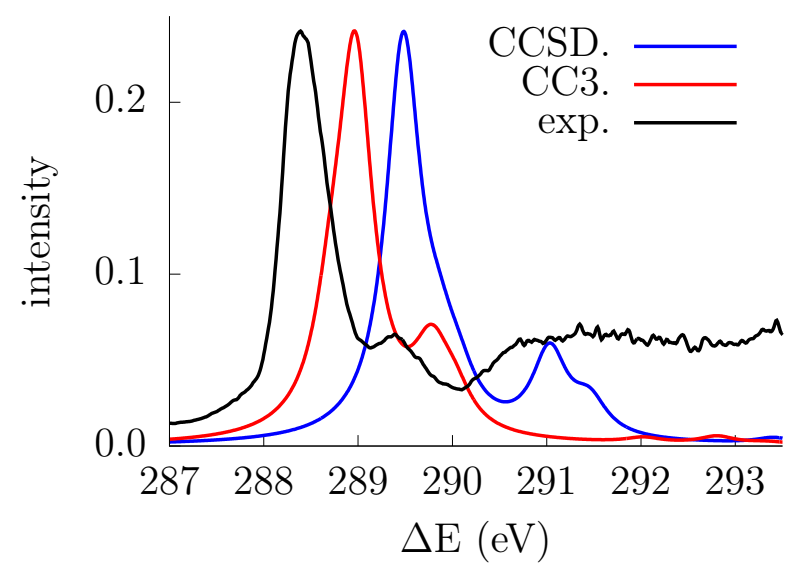

(a) Unshifted

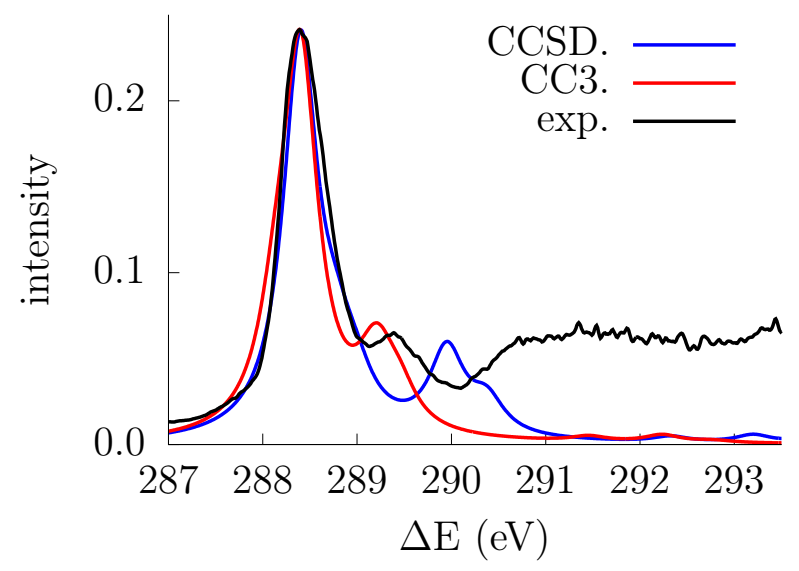

(b) Shifted

Figure 3: Experimental and simulated carbon K-edge spectra of conformer I. The simulated spectra are constructed using CCSD and CC3 excitation energies and CCSD transition strengths with the $\mathrm{a}(\mathrm{CT}) \mathrm{Z}$ basis set. In panel $3 \mathrm{a}$, the theoretical spectrum is unshifted while the peaks are shifted to fit the most intense peak of $\mathrm{C} 2$ in panel 3b. CCSD shift: $-1.08 \mathrm{eV}$. CC3 shift: $-0.57 \mathrm{eV}$.

Note that the theoretical spectra are simulated using CCSD and CC3 excitation energies and CCSD transition strengths. For the lowest excitation, the CCSD and CC3 excitation 
vectors are similar and the CCSD transition strengths are a reasonable approximation to the CC3 strengths. However, for the higher states, the differences tend to increase and CC3 transition strengths should be used. Unfortunately, we do not have CVS-CC3 transition strengths available yet.

For $\mathrm{C} 1$, we observe the largest absolute error between $\mathrm{CC} 3 / \mathrm{a}(\mathrm{CQ}) \mathrm{Z}$ and the experimental assignments at almost $0.9 \mathrm{eV}$. In this case, we suspect the experimental assignment is incorrect. The carbon spectra are presented in Figure 3, and they are dominated by the intense peak from $\mathrm{C} 2$, and the $\mathrm{C} 1$ edge only appears as a shoulder on the main peak. Plekan et al. used the STEX method to interpret the experimental results, but it appears that this method is not accurate enough and misses several states that are obtained with CCSD and CC3. The $\mathrm{CC} 3$ results indicates that there are two excitations from $\mathrm{C} 1$ in the area, both contributing intensity to the main peak. Furthermore, the second $\mathrm{A}^{\prime \prime}$ excitation calculated with $\mathrm{CC} 3 / \mathrm{a}(\mathrm{CT}) \mathrm{Z}$ is only $0.39 \mathrm{eV}$ too high and would likely be lowered with a larger basis set.

There is only one experimental peak assigned to $\mathrm{C} 2$ and the absolute error for the first excitation is $0.38 \mathrm{eV}$ with $\mathrm{CC} 3 / \mathrm{a}(\mathrm{CQ}) \mathrm{Z}$. Again, there is some uncertainty in the assignment of the experimental peak because the mixing with the $\mathrm{C} 1$ signals may move the apparent peak down, increasing the error for CC3. In carbon monoxide, the first core excitation of $\mathrm{C}$ is about $0.2 \mathrm{eV}$ too high and CCSDT is required to lower it further ${ }^{45}$ so we would expect CC3 to be too high in this case as well.

The experimental and simulated X-ray spectra at the nitrogen K-edge are presented in Figure 4. Besides the absolute error, $0.14 \mathrm{eV}$ for $\mathrm{CC} 3,1.70 \mathrm{eV}$ for CCSD, the spectra look quite similar, except that the CC3 spectrum is more compressed. Due to the greater overlaps between the signals in the main peak and normalization, the other peaks appear less intense in CC3. For the fourth excitation, the relative position is about $0.4 \mathrm{eV}$ lower in CC3 than in CCSD. Comparing the simulated and experimental spectra, it appears that the CC3 peak is between the experimental peaks and it is difficult to say where it should 


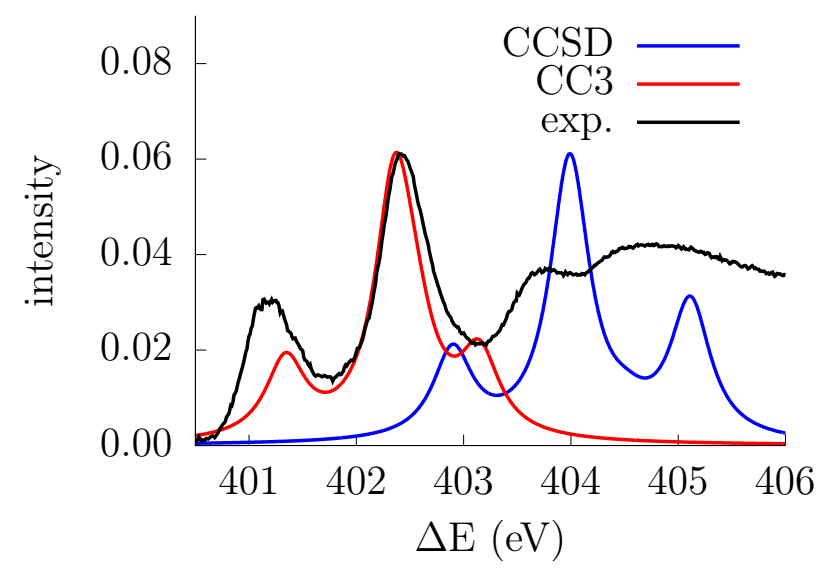

(a) Unshifted

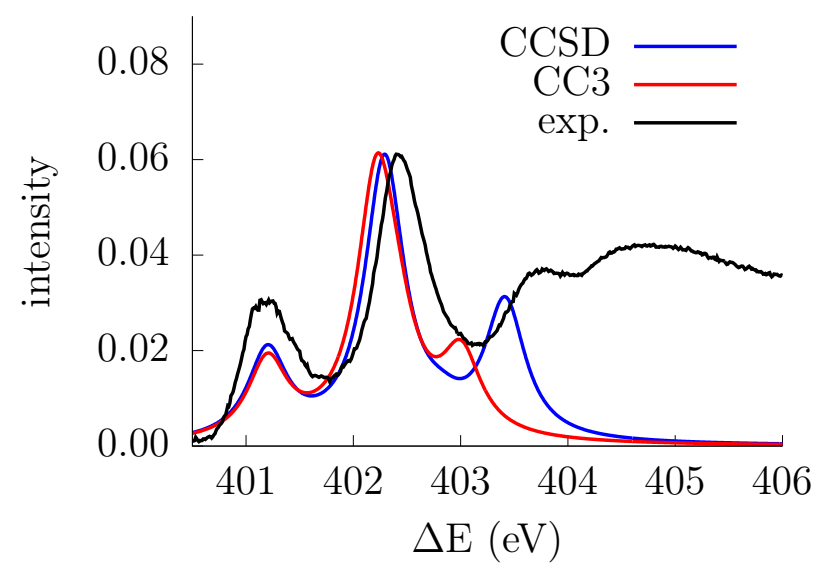

(b) Shifted

Figure 4: Experimental and simulated nitrogen K-edge spectrum of conformer I. The simulated spectrum is constructed using CCSD and CC3 excitation energies and CCSD transition moments with the a(CT)Z basis set. In panel $4 \mathrm{a}$ the theoretical spectrum is unshifted, whereas the peaks are shifted to fit the nitrogen K-edge in panel 4b. CCSD shift: $(-1.70$ $\mathrm{eV})$. CC3 shift: $(-0.14 \mathrm{eV})$. 
be. There are several possible reasons for this, for example, the intensities are calculated at the CCSD level, but there are considerable differences between the CCSD and CC3 vectors. In ammonia, ${ }^{45}$ the differences between CCSD and CC3 are relatively small, with CCSD tending to overestimate the relative excitation energies and CC3 underestimating, but much less than what is observed here for glycine. Notably, including extra diffuse basis functions on ammonia reduces the relative positions of the higher states with Rydberg character 0.2 to $0.3 \mathrm{eV}$. A similar shift for the fifth $\mathrm{CC} 3$ excitation in glycine would move it into the main peak.

Finally, the oxygen spectra are presented in Figure 5. For O4 the main feature visible in the spectrum is the strong peak at $532.2 \mathrm{eV}$ from the double bonded oxygen to the $\mathrm{CO}$ $\pi$ orbital. CC3 slightly underestimates the excitation energy and larger basis sets appear to lower it further, but the CC3 shift between Figures 5a and 5b is only $0.10 \mathrm{eV}$. While CCSD indicates that there is some intensity in the higher transitions, this mostly disappears behind the excitations from O5. Again we can compare to CO, where CC3 somewhat underestimates the excitation energy while CCSDT is a bit higher. ${ }^{45}$

For $\mathrm{O} 5$, the most visible feature of the simulated spectrum is the peak at $535.2 \mathrm{eV}$ tha is too intense. The intensity is calculated using CCSD and it might be reduced in a CC3, but the main reason is probably that the two excited states are nearly degenerate when using $\mathrm{CC} 3 / \mathrm{a}(\mathrm{CT}) \mathrm{Z}$. This results in a too high and narrow peak. It is possible that the states would be further apart in a larger basis set, because the $\mathrm{A}^{\prime \prime}$ excitation goes to the $\pi$ orbital which is more delocalized, see Table 2. Similarly to nitrogen, it appears that the higher peaks are too close. However, again there are quite large differences between the CCSD and CC3 vectors and the calculated intensities may be different as well. These excitations are also so high in energy that additional Rydberg functions may be required to describe them.

Core ionization energies are compared with experiment in Table 6. The overall pattern is similar to the core excitation energies, with the calculated energies reduced by about 1.5-3.0 $\mathrm{eV}$ when going from CCSD to CC3 and reductions of 1.0-2.0 eV when increasing the basis 


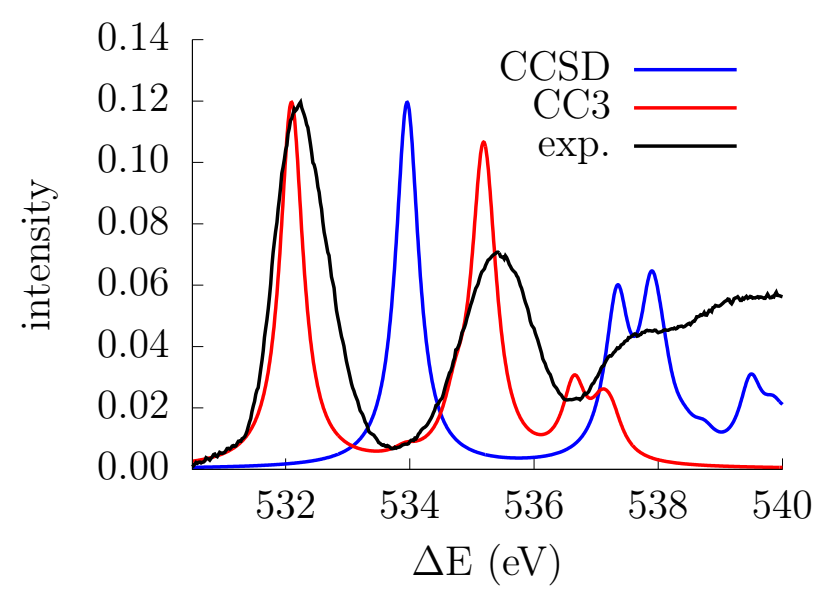

(a) Unshifted

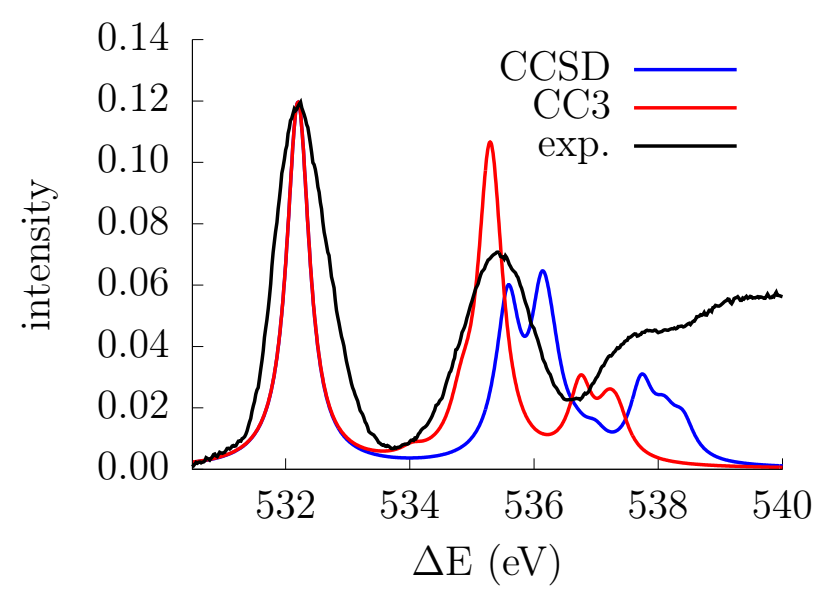

(b) Shifted

Figure 5: Experimental and simulated oxygen K-edge spectra of conformer I. The simulated spectrum is constructed using CCSD and CC3 excitation energies and CCSD transition strengths with the a $(\mathrm{CT}) \mathrm{Z}$ basis set. In panel $5 \mathrm{a}$, the theoretical spectrum is unshifted while the peaks are shifted to fit the K-edge of the corresponding atom in panel 5b. CCSD shift: $-1.76 \mathrm{eV}, \mathrm{CC} 3 \mathrm{shift}:+0.10 \mathrm{eV}$. 
from aCDZ to a(CT)Z. The largest dependence on basis and model is observed for oxygen, again similar to the case of the excitation energies.

For the excitation energies, CC3 was very close to the experimental K-edge for nitrogen, while too high for carbon and too low for oxygen. For the ionizations, it is carbon that is closest to experiment, but somewhat too low, but the pattern is the same with calculated results being lower compared to experiment for the heavier cores. Relativistic effects can partially explain this pattern, because scalar relativistic effects increase excitation energies from heavier cores more than those from lighter cores.

Another factor missing in these calculations is vibrational effects. These would broaden the peaks because transitions between different vibrational states would give different energies. In addition, vibrational effects might move the average position of the peak up if transitions to higher vibrational states are relatively strong. ${ }^{44}$

Table 6: Conformer I. CCSD and CC3 core ionization energies and comparison with the experimental results from Plekan et al. ${ }^{12}(\mathrm{eV})$.

\begin{tabular}{lllllll}
\hline & & $\mathrm{aCDZ}$ & $\mathrm{a}(\mathrm{CT}) \mathrm{Z}$ & $\mathrm{aCTZ}$ & $\mathrm{a}(\mathrm{CQ}) \mathrm{Z}$ & exp. $( \pm 0.1)$ \\
\hline \multirow{2}{*}{ C1 } & CCSD & 294.68 & 293.75 & 293.79 & 293.70 & \multirow{2}{*}{292.3} \\
& CC3 & 293.37 & 292.35 & 292.40 & 292.23 & \\
$\mathrm{C} 2$ & CCSD & 297.92 & 296.98 & 297.04 & 296.93 & \multirow{2}{*}{295.2} \\
& CC3 & 296.57 & 295.52 & 295.58 & 295.39 & \\
N3 & CCSD & 408.56 & 407.14 & 407.17 & 407.08 & \multirow{2}{*}{405.4} \\
& CC3 & 406.48 & 405.09 & 405.11 & 404.95 & \\
O4 & CCSD & 542.71 & 540.75 & 540.81 & 540.66 & 5 \\
& CC3 & 538.91 & 537.24 & 537.29 & 537.09 & \\
O55 & CCSD & 544.22 & 542.33 & 542.38 & 542.24 & \multirow{2}{*}{540.2} \\
& CC3 & 540.83 & 539.21 & 539.24 & 539.05 & \\
\hline
\end{tabular}

\section{Effect of conformers}

In Table 7, we present core excitation energies and oscillator strengths of O5 for the different conformers, calculated using the aCDZ basis set. Only small differences in the excitation energies and oscillator strengths are observed between the different conformers, except for con- 
former II. For this conformer, we predict that the lowest excitation has $\mathrm{A}^{\prime \prime}$ symmetry and is considerably more intense than the lowest $\mathrm{A}^{\prime}$ excitation. Boltzmann weights were calculated based on total electronic energies and zero point vibrational energies at the $\operatorname{CCSD}(\mathrm{T}) /$ augcc-pVDZ level. The weight of conformer I is 0.73 , while the weight of conformer II is 0.13 and the rest considerably lower at 298K. Figure 6 compares the averaged oxygen spectrum with that of conformer I. There is a slight broadening of the peaks in Figure 6b compared to $6 \mathrm{a}$, but this would be very hard to detect experimentally. ${ }^{11}$

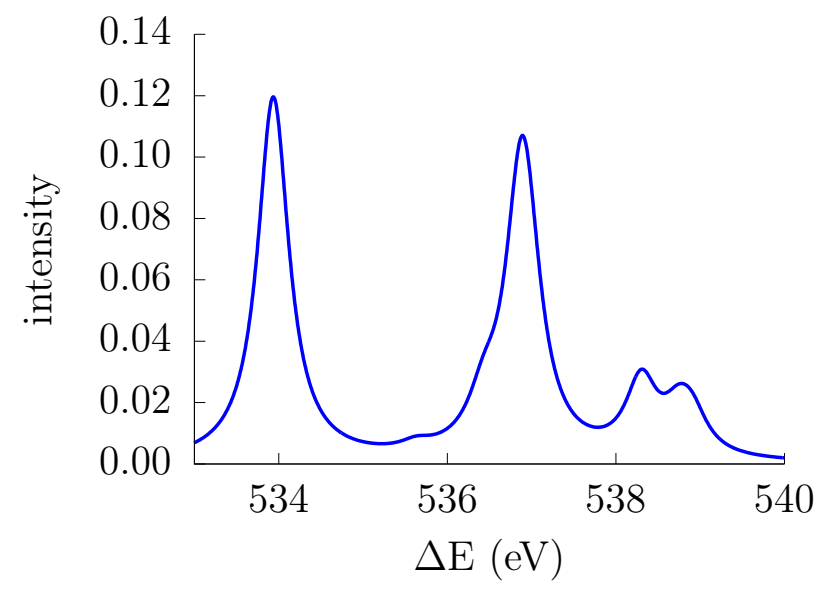

(a) Conformer I

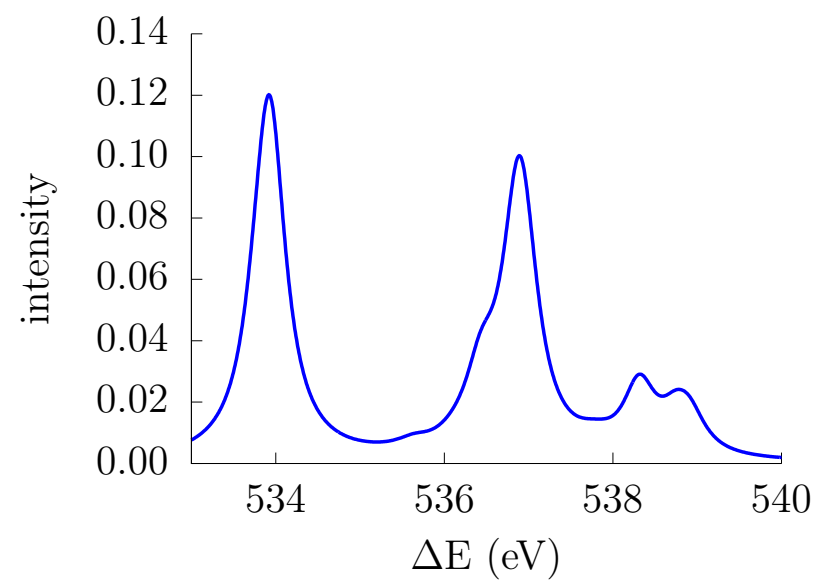

(b) Averaged

Figure 6: Oxygen spectra of glycine calculated using five CC3/aCDZ excitations for each atom at the and CCSD/aCDZ transition moments. In the upper panel, the calculated spectrum for Conformer I is shown, while the lower panel shows the Boltzmann-weighted spectrum of five conformers at $298 \mathrm{~K}$.

Using CC3, the peaks in the spectrum move closer together, see Table 8, and the $\mathrm{A}^{\prime \prime}$ 
Table 7: Glycine. CCSD/aCDZ core excitations (eV) and intensities for O5.

\begin{tabular}{|c|c|c|c|c|c|c|c|c|c|c|c|c|}
\hline \multicolumn{3}{|c|}{ I } & \multicolumn{3}{|c|}{ II } & \multicolumn{3}{|c|}{ III } & \multicolumn{2}{|c|}{ IV } & \multicolumn{2}{|c|}{ V } \\
\hline$\Delta \mathrm{E}$ & $f$ & Sym. & $\Delta \mathrm{E}$ & $f$ & Sym. & $\Delta \mathrm{E}$ & $f$ & Sym. & $\Delta \mathrm{E}$ & $f$ & $\Delta \mathrm{E}$ & $f$ \\
\hline 539.19 & 1.56 & $\mathrm{~A}^{\prime}$ & 539.17 & 1.90 & $\mathrm{~A}^{\prime \prime}$ & 539.23 & 1.51 & $\mathrm{~A}^{\prime}$ & 539.23 & 1.58 & 539.21 & 1.53 \\
\hline+0.55 & 1.46 & $\mathrm{~A}^{\prime \prime}$ & +0.46 & 0.47 & $\mathrm{~A}^{\prime}$ & +0.39 & 1.66 & $\mathrm{~A}^{\prime \prime}$ & +0.50 & 1.49 & +0.47 & 1.50 \\
\hline+2.18 & 0.76 & $\mathrm{~A}^{\prime}$ & +1.88 & 0.36 & $\mathrm{~A}^{\prime}$ & +2.02 & 1.04 & $\mathrm{~A}^{\prime}$ & +2.20 & 0.77 & +2.10 & 0.89 \\
\hline+2.55 & 0.37 & $\mathrm{~A}^{\prime \prime}$ & +2.06 & 0.15 & $A^{\prime \prime}$ & +2.57 & 0.28 & $A^{\prime \prime}$ & +2.62 & 0.41 & +2.65 & 0.29 \\
\hline+2.85 & 0.36 & $\mathrm{~A}^{\prime}$ & +2.15 & 0.27 & $\mathrm{~A}^{\prime}$ & +2.80 & 0.38 & $\mathrm{~A}^{\prime}$ & +2.97 & 0.30 & +2.99 & 0.41 \\
\hline
\end{tabular}

state is the lowest for all three conformers. Conformer II is $0.4 \mathrm{eV}$ lower than the others, but again, this would be hard to resolve in an experiment. For the other atoms, smaller differences are observed between the conformers, see Supplementary Information.

Table 8: Glycine. CC3/aCDZ core excitations of $\mathrm{O} 5(\mathrm{eV})$.

\begin{tabular}{rlrlrlrc}
\hline $\mathrm{I}$ & & $\mathrm{II}$ & & $\mathrm{III}$ & $\mathrm{IV}$ & $\mathrm{V}$ \\
\hline 536.86 & $\mathrm{~A}^{\prime \prime}$ & 536.44 & $\mathrm{~A}^{\prime \prime}$ & 536.84 & $\mathrm{~A}^{\prime \prime}$ & 536.86 & 536.78 \\
+0.06 & $\mathrm{~A}^{\prime}$ & +0.21 & $\mathrm{~A}^{\prime}$ & +0.08 & $\mathrm{~A}^{\prime}$ & +0.13 & +0.18 \\
+1.44 & $\mathrm{~A}^{\prime}$ & +1.29 & $\mathrm{~A}^{\prime}$ & +1.51 & $\mathrm{~A}^{\prime}$ & +1.55 & +1.60 \\
+1.88 & $\mathrm{~A}^{\prime \prime}$ & +1.39 & $\mathrm{~A}^{\prime \prime}$ & +1.91 & $\mathrm{~A}^{\prime \prime}$ & +2.05 & +1.98 \\
+2.04 & $\mathrm{~A}^{\prime}$ & +1.41 & $\mathrm{~A}^{\prime}$ & +1.99 & $\mathrm{~A}^{\prime}$ & +2.15 & +2.21 \\
\hline
\end{tabular}




\section{Conclusions}

In this study, we have simulated UV and X-ray spectra of glycine using CCSD and CC3. The X-ray spectra have then been compared to experiment ${ }^{11}$ and we have reinterpreted some of the experimental assignments. ${ }^{11}$ In particular, the coupled cluster methods indicate that the main peaks in the nitrogen and carbon NEXAFS spectra are caused by three different transitions while the STEX method ${ }^{11}$ indicates it is only one.

Core excitations exhibit a strong dependence on the basis set, with shifts of more than $1 \mathrm{eV}$ when increasing the basis set from aCDZ to a $(\mathrm{CT}) \mathrm{Z}$ on the core being excited. More modest shifts are observed when increasing the basis further, so the a $(\mathrm{CT}) \mathrm{Z}$ basis is sufficient to get absolute errors less than $1 \mathrm{eV}$ with $\mathrm{CC} 3$. The changes in the difference between excitation energies and transition moments are much smaller when changing basis. However, these changes appear to be somewhat larger for the double bonded atoms. It should be noted that extra diffuse functions, that have not been included here, significantly change the

relative positions of the higher excitations in small molecules ${ }^{45,47}$ by reducing the excitation energy.

The main differences between CCSD and CC3 are large changes in the absolute excitation energies of 1-2 eV. In addition, the relative excitation energies are significantly reduced with CC3 and for C1 the order of the states changes.

Because of the overlapping states, it is difficult to compare the models to experiment without CC3 intensities, but CC3 appears to give better agreement for oxygen and carbon at least. We have also modeled the effect of the different conformers of glycine on the NEXAFS spectra. However, the differences between the conformers are predicted to be small and the lowest energy conformer dominates. Consequently, we do not expect to see much of an effect besides a slight broadening of the peaks at room temperature. 


\section{Acknowledgement}

We are grateful to Kevin Prince for providing us with the data for the experimental NEXAFS spectra. S.C. thanks Cristina Puzzarini for discussion. R.H.M acknowledges financial support through ERC-STG-2014 under grant No 639508 and from the Research Council of Norway through its Centres of Excellence scheme, project number 262695. H.K. acknowledges financial support from the FP7-PEOPLE-2013-IOF funding scheme (Project No. 625321). S.C. acknowledges financial support from the AIAS-COFUND program (Grant Agreement No. 609033) and from the Independent Research Fund Denmark, DFFFNU Research Project 2 No. 7014-00258B. The COST Actions No. CM1002, "COnvergent Distributed Environment for Computational Spectroscopy (CODECS)", MP1306, "Modern Tools for Spectroscopy on Advanced Materials (EUSPEC)", and CM1204, "XUV/X-ray light and fast ions for ultrafast chemistry (XLIC)", are also acknowledged. We acknowledge computer time from NOTUR through Project No. nn2962k and nn4654k. S.C. and H. K. are part of the Marie Skłodowska-Curie European Training Network "COSINE - COmputational Spectroscopy In Natural sciences and Engineering", Grant Agreement No. 765739.

\section{Supporting Information Available}

Molecular geometries used in the calculations, zero point energies and calculated excitation energies and oscillator strengths are available as additional information.

\section{References}

(1) Miller, III, T. F.; Clary, D. C. Quantum free energies of the conformers of glycine on an ab initio potential energy surface. Phys. Chem. Chem. Phys. 2004, 6, 2563-2571.

(2) Kuan, Y.-J.; Charnley, S. B.; Huang, H.-C.; Tseng, W.-L.; Kisielo, Z. Interstellar Glycine. Astrophys. J. 2003, 593, 848-867. 
(3) Snyder, L. E.; Lovas, F. J.; Hollis, J. M.; Friedel, D. N.; Jewell, P. R.; Remijan, A.; Ilyushin, V. V.; Alekseev, E. A.; Dyubko, S. F. A Rigorous Attempt to Verify Interstellar Glycine. Astrophys. J. 2005, 619, 914.

(4) Jones, P. A.; Cunningham, M. R.; Godfrey, P. D.; Cragg, D. M. A Search for biomolecules in Sagittarius B2 (LMH) with the Australia Telescope Compact Array. Mon. Notices Royal Astron. Soc. 2006, 374, 579-589.

(5) Cunningham, M. R.; Jones, P. A.; Godfrey, P. D.; Cragg, D. M.; Bains, I.; Burton, M. G.; Calisse, P.; Crighton, N. H. M.; Curran, S. J.; Davis, T. M. et al. A search for propylene oxide and glycine in Sagittarius B2 (LMH) and Orion. Mon. Notices Royal Astron. Soc. 2007, 376, 1201-1210.

(6) Lattelais, M.; Pauzat, F.; Pilmé, J.; Ellinger, Y.; Ceccarelli, C. About the detectability of glycine in the interstellar medium. A\&A 2011, 532, A39.

(7) Barone, V.; Biczysko, M.; Bloino, J.; Puzzarini, C. Glycine conformers: a never-ending story? Phys. Chem. Chem. Phys. 2013, 15, 1358-1363.

(8) Barone, V.; Biczysko, M.; Bloino, J.; Puzzarini, C. Characterization of the Elusive Conformers of Glycine from State-of-the-Art Structural, Thermodynamic, and Spectroscopic Computations: Theory Complements Experiment. J. Chem. Theory Comput. 2013, 9, 1533-1547.

(9) Barone, V.; Biczysko, M.; Bloino, J.; Puzzarini, C. The performance of composite schemes and hybrid CC/DFT model in predicting structure, thermodynamic and spectroscopic parameters: the challenge of the conformational equilibrium in glycine. Phys. Chem. Chem. Phys. 2013, 15, 10094-10111.

(10) Gordon, M. L.; Cooper, G.; Morin, C.; Araki, T.; Turci, C. C.; Kaznatcheev, K.; Hitchcock, A. P. Inner-Shell Excitation Spectroscopy of the Peptide Bond: Comparison 
of the C 1s, N 1s, and O 1s Spectra of Glycine, Glycyl-Glycine, and Glycyl-GlycylGlycine. J. Phys. Chem. A 2003, 10\%, 6144-6159.

(11) Plekan, O.; Feyer, V.; Richter, R.; Coreno, M.; de Simone, M.; Prince, K.; Carravetta, V. An X-ray absorption study of glycine, methionine and proline. J. Electron Spectrosc. Relat. Phenom. 2007, 155, $47-53$.

(12) Plekan, O.; Feyer, V.; Richter, R.; Coreno, M.; de Simone, M.; Prince, K. C.; Carravetta, V. Investigation of the Amino Acids Glycine, Proline, and Methionine by Photoemission Spectroscopy. J. Phys. Chem. A 2007, 111, 10998-11005.

(13) Messer, B. M.; Cappa, C. D.; Smith, J. D.; Wilson, K. R.; Gilles, M. K.; Cohen, R. C.; Saykally, R. J. pH Dependence of the Electronic Structure of Glycine. J. Phys. Chem. B 2005, 109, 5375-5382.

(14) Kaznacheyev, K.; Osanna, A.; Jacobsen, C.; Plashkevych, O.; Vahtras, O.; H.Ågren,; Carravetta, V.; Hitchcock, A. P. Innershell Absorption Spectroscopy of Amino Acids. J. Phys. Chem. A 2002, 106, 3153-3168.

(15) Zubavichus, Y.; Zharnikov, M.; Schaporenko, A.; Grunze, M. NEXAFS study of glycine and glycine-based oligopeptides. J. Electron Spectrosc. Relat. Phenom. 2004, 134, 25 -33 .

(16) Norman, P.; Dreuw, A. Simulating X-ray Spectroscopies and Calculating Core-Excited States of Molecules. Chem. Rev. 2018, 118, 7208-7248.

(17) Ågren, H.; Carravetta, V.; Vahtras, O.; Pettersson, M. L. G. Direct SCF direct staticexchange calculations of electronic spectra. Theor. Chem. Acc. 1997, 97, 14-40.

(18) Schirmer, J. Beyond the random-phase approximation: A new approximation scheme for the polarization propagator. Phys. Rev. A 1982, 26, 2395-2416. 
(19) Dreuw, A.; Wormit, M. The algebraic diagrammatic construction scheme for the polarization propagator for the calculation of excited states. WIREs Comput. Mol. Sci. 2015, 5, 82-95.

(20) Cederbaum, L. S.; Domcke, W.; Schirmer, J. Many-body theory of core holes. Phys. Rev. A 1980, 22, 206-222.

(21) Barth, A.; Schirmer, J. Theoretical core-level excitation spectra of $\mathrm{N}_{2}$ and CO by a new polarisation propagator method. J. Phys. B: At. Mol. Phys. 1985, 18, 867.

(22) Trofimov, A. B.; Moskovskaya, T. É.; Gromov, E. V.; Vitkovskaya, N. M.; Schirmer, J. Core-level electronic spectra in $\mathrm{ADC}(2)$ approximation for polarization propagator: carbon monoxide and nitrogen molecules. J. Struct. Chem. 2000, 41, 483-494.

(23) Wenzel, J.; Wormit, M.; Dreuw, A. Calculating core-level excitations and X-ray absorption spectra of medium-sized closed-shell molecules with the algebraic-diagrammatic construction scheme for the polarization propagator. J. Comput. Chem. 2014, 35, 19001915.

(24) Wenzel, J.; Wormit, M.; Dreuw, A. Calculating X-ray Absorption Spectra of Open-Shell Molecules with the Unrestricted Algebraic-Diagrammatic Construction Scheme for the Polarization Propagator. J. Chem. Theory Comput. 2014, 10, 4583-4598.

(25) Č́žžek, J. On the Correlation Problem in Atomic and Molecular Systems. Calculation of Wavefunction Components in Ursell-Type Expansion Using Quantum-Field Theoretical Methods. J. Chem. Phys. 1966, 45, 4256-4266.

(26) Paldus, J.; Č́̌žek, J.; Shavitt, I. Correlation Problems in Atomic and Molecular Systems. IV. Extended Coupled-Pair Many-Electron Theory and Its Application to the $\mathrm{BH}_{3}$ Molecule. Phys. Rev. A 1972, 5, 50-67. 
(27) Purvis, G. D.; Bartlett, R. J. A full coupled-cluster singles and doubles model: The inclusion of disconnected triples. J. Chem. Phys. 1982, 76, 1910-1918.

(28) Koch, H.; Christiansen, O.; Jørgensen, P.; Sánchez de Merás, A. M.; Helgaker, T. The CC3 model: An iterative coupled cluster approach including connected triples. J. Chem. Phys. 1997, 106, 1808-1818.

(29) Koch, H.; Jørgensen, P. Coupled cluster response functions. J. Chem. Phys. 1990, 93, 3333-3344.

(30) Pedersen, T. B.; Koch, H. Coupled cluster response functions revisited. J. Chem. Phys. 1997, 106, 8059-8072.

(31) Rowe, D. J. Equations-of-Motion Method and the Extended Shell Model. Rev. Mod. Phys. 1968, 40, 153-166.

(32) Stanton, J. F.; Bartlett, R. J. The equation of motion coupled-cluster method. A systematic biorthogonal approach to molecular excitation energies, transition probabilities, and excited state properties. J. Chem. Phys. 1993, 98, 7029-7039.

(33) Nooijen, M.; Bartlett, R. J. Description of core-excitation spectra by the open-shell electron-attachment equation-of-motion coupled cluster method. J. Chem. Phys. 1995, 102, 6735-6756.

(34) Coriani, S.; Fransson, T.; Christiansen, O.; Norman, P. Asymmetric-Lanczos-ChainDriven Implementation of Electronic Resonance Convergent Coupled-Cluster Linear Response Theory. J Chem. Theory Comput. 2012, 8, 1616-1628.

(35) Coriani, S.; Christiansen, O.; Fransson, T.; Norman, P. Coupled-cluster response theory for near-edge X-ray-absorption fine structure of atoms and molecules. Phys. Rev. A 2012, 85, 022507. 
(36) Southworth, S. H.; Wehlitz, R.; Picón, A.; Lehmann, C. S.; Cheng, L.; Stanton, J. F. Inner-shell photoionization and core-hole decay of $\mathrm{Xe}$ and $\mathrm{XeF}_{2}$. J. Chem. Phys. 2015, $142,224302$.

(37) Brabec, J.; Bhaskaran-Nair, K.; Govind, N.; Pittner, J.; Kowalski, K. Communication: Application of state-specific multireference coupled cluster methods to core-level excitations. J. Chem. Phys. 2012, 13\%, 171101.

(38) Kauczor, J.; Norman, P.; Christiansen, O.; Coriani, S. Communication: A reducedspace algorithm for the solution of the complex linear response equations used in coupled cluster damped response theory. J. Chem. Phys. 2013, 139, 211102.

(39) Peng, B.; Lestrange, P. J.; Goings, J. J.; Caricato, M.; Li, X. Energy-Specific Equationof-Motion Coupled-Cluster Methods for High-Energy Excited States: Application to K-edge X-ray Absorption Spectroscopy. J. Chem. Theory Comp. 2015, 11, 4146-4153.

(40) Nascimento, D. R.; DePrince III, A. E. Simulation of Near-Edge X-ray Absorption Fine Structure with Time-Dependent Equation-of-Motion Coupled-Cluster Theory. J. Phys. Chem. Lett. 2017, 8, 2951-2957.

(41) Coriani, S.; Koch, H. Communication: X-ray absorption spectra and core-ionization potentials within a core-valence separated coupled cluster framework. J. Chem. Phys. 2015, 143, 181103.

(42) Coriani, S.; Koch, H. Erratum: "Communication: X-ray absorption spectra and coreionization potentials within a core-valence separated coupled cluster framework" J. Chem. Phys. 143, 181103 (2015). J. Chem. Phys. 2016, 145, 149901.

(43) Wolf, T. J. A.; Myhre, R. H.; Cryan, J. P.; Coriani, S.; Battistoni, A.; Berrah, N.; Bostedt, C.; Bucksbaum, P.; Coffee, R. N.; Coslovich, G. et al. Probing ultrafast $\pi \pi^{*} / \mathrm{n} \pi^{*}$ internal conversion in organic chromophores via K-edge resonant absorption. Nat. Comm. 2017, 8, 2041-1723. 
(44) Myhre, R. H.; Wolf, T. J. A.; Cheng, L.; Nandi, S.; Coriani, S.; Gühr, M.; Koch, H. A theoretical and experimental benchmark study of core-excited states in nitrogen. $J$. Chem. Phys. 2018, 148, 064106.

(45) Carbone, J. P.; Cheng, L.; Myhre, R. H.; Matthews, D.; Koch, H.; Coriani, S. An analysis of the performance of core-valence separated coupled cluster methods for core excitations and core ionizations using standard basis sets. Adv. Quantum Chem. 2019,

(46) Liu, J.; Matthews, D.; Coriani, S.; Cheng, L. Benchmark Calculations of K-Edge Ionization Energies for First-Row Elements Using Scalar-Relativistic Core-Valence-Separated Equation-of-Motion Coupled-Cluster Methods. J. Chem. Theory Comput. 2019, 15, $1642-1651$.

(47) Frati, F.; de Groot, F.; Cerezo, J.; Santoro, F.; Cheng, L.; Faber, R.; Coriani, S. Coupled cluster study of the X-ray absorption spectra of formaldehyde derivatives at the oxygen, carbon, and fluorine K-edges. J. Chem. Phys. 2019, 151, 064107.

(48) Vidal, M. L.; Feng, X.; Epifanovsky, E.; Krylov, A. I.; Coriani, S. New and Efficient Equation-of-Motion Coupled-Cluster Framework for Core-Excited and Core-Ionized States. J. Chem. Theory Comput. 2019, 15, 3117-3133.

(49) Myhre, R. H.; Sánchez de Merás, A. M. J.; Koch, H. Multi-level coupled cluster theory. J. Chem. Phys. 2014, 141,- .

(50) Myhre, R. H.; Koch, H. The multilevel CC3 coupled cluster model. J. Chem. Phys. 2016, 145 .

(51) Myhre, R. H.; Coriani, S.; Koch, H. Near-Edge X-ray Absorption Fine Structure within Multilevel Coupled Cluster Theory. J. Chem. Theory Comput. 2016, 12, 2633-2643.

(52) Høyvik, I.-M.; Myhre, R. H.; Koch, H. Correlated natural transition orbitals for core 
excitation energies in multilevel coupled cluster models. J. Chem. Phys. 2017, 146, 144109.

(53) Sadybekov, A.; Krylov, A. I. Coupled-cluster based approach for core-level states in condensed phase: Theory and application to different protonated forms of aqueous glycine. J. Chem. Phys. 2017, 147, 014107.

(54) Zheng, X.; Cheng, L. Performance of Delta-Coupled-Cluster Methods for Calculations of Core-Ionization Energies of First-Row Elements. J. Chem. Theory Comput. 2019,

(55) Davidson, E. J. The Iterative Calculation of a Few of the Lowest Eigenvalues and Corresponding Eigenvectors of Large Real-Symmetric Matrices. J. Comp. Phys. 1975, $17,87-94$.

(56) Stanton, J. F.; Gauss, J. A simple scheme for the direct calculation of ionization potentials with coupled-cluster theory that exploits established excitation energy methods. J. Chem. Phys. 1999, 111, 8785-8788.

(57) Arneberg, R.; Müller, J.; Manne, R. Configuration interaction calculations of satellite structure in photoelectron spectra of H2O. Chem. Phys. 1982, 64, $249-258$.

(58) Melania Oana, C.; Krylov, A. I. Dyson orbitals for ionization from the ground and electronically excited states within equation-of-motion coupled-cluster formalism: Theory, implementation, and examples. J. Chem. Phys. 2007, 127.

(59) Stanton, J.; Gauss, J.; Harding, M.; Szalay, P.; Auer, A.; Bartlett, R.; Benedikt, U.; Berger, C.; Bernholdt, D.; Bomble, Y. et al. CFOUR, Coupled-Cluster techniques for Computational Chemistry http://www.cfour.de. 2015; http://www.cfour.de/.

(60) Harding, M. E.; Metzroth, T.; Gauss, J.; Auer, A. A. Parallel Calculation of CCSD and CCSD(T) Analytic First and Second Derivatives. J. Chem. Theory Comput. 2008, 4, $64-74$. 
(61) Kendall, R. A.; Dunning, T. H.; Harrison, R. J. Electron affinities of the first-row atoms revisited. Systematic basis sets and wave functions. J. Chem. Phys. 1992, 96, 6796-6806.

(62) Helgaker, T.; Jørgensen, P.; Olsen, J. Molecular Electronic-Structure Theory; John Wiley \& Sons, LTD: The Atrium, Southern Gate, Chichester, West Sussex, PO19 8SQ, England, 2004; Chapter Calibration of the Electronic-Structure Models, pp 821-836.

(63) Gauss, J.; Stanton, J. F. Analytic $\operatorname{CCSD}(\mathrm{T})$ second derivatives. Chem. Phys. Lett. 1997, $276,70-77$.

(64) Woon, D. E.; Dunning, T. H. Gaussian basis sets for use in correlated molecular calculations. V. Core-valence basis sets for boron through neon. J. Chem. Phys. 1995, 103, $4572-4585$.

(65) Osted, A.; Kongsted, J.; Christiansen, O. Theoretical Study of the Electronic GasPhase Spectrum of Glycine, Alanine, and Related Amines and Carboxylic Acids. J. Phys. Chem. A 2005, 109, 1430-1440. 
Graphical TOC Entry

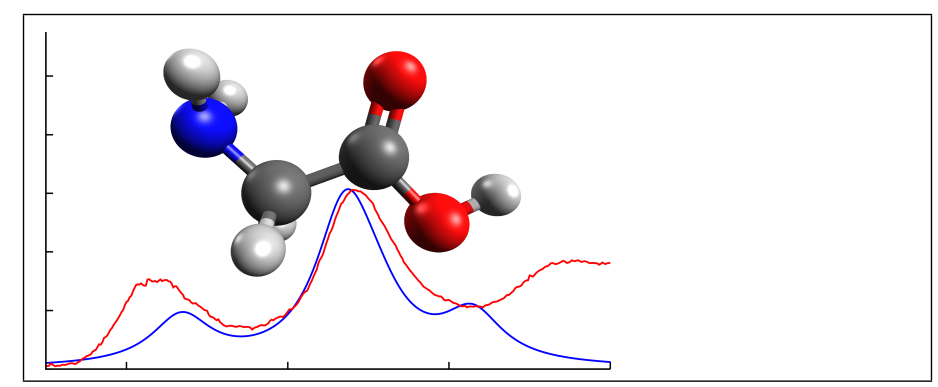

